\title{
Evaluation of Wrinkling States Using In-Process Ultrasonic Examination during Sheet Metal Forming*1
}

\author{
Ryota Kakinoki ${ }^{1, * 2}$, Yuji Segawa ${ }^{2, * 3}$, Yasuo Marumo ${ }^{3}$, Yasuhiro Imamura ${ }^{4}$, \\ Tomohiro Nonaka ${ }^{5}$ and Yutaka Sakata ${ }^{5}$ \\ ${ }^{1}$ Graduate School of Science and Technology, Kumamoto University, Kumamoto 860-8555, Japan \\ ${ }^{2}$ Department of Mechanical Engineering, National Institute of Technology, Miyakonojo College, Miyakonojo 860-8555, Japan \\ ${ }^{3}$ Faculty of Advanced Science and Technology, Kumamoto University, Kumamoto 860-8555, Japan \\ ${ }^{4}$ Technical Division, Faculty of Engineering, Kumamoto University, Kumamoto 860-8555, Japan \\ ${ }^{5}$ Department of Integrated System Engineering, Nishinippon Institute of Technology, Fukuoka 800-0394, Japan
}

\begin{abstract}
In press forming, sampling inspection has been performed during production. However, in sampling inspection, there is the possibility that defective products will proceed to the next process despite inspection. In this paper, we investigated a method for detecting wrinkle defects occurring during deep drawing in order to prevent the outflow of defective products. Deep-drawing tests were performed using a drawing die incorporating an ultrasonic transducer. During deep drawing, ultrasonic waves were irradiated to the interface between the die and the workpiece, and the occurrence of wrinkles was examined on the basis of ultrasonic reflection characteristics. The ultrasonic reflection intensity was changed by the occurrence of the wrinkles. The influence of wrinkle height and wrinkle wavelength on relative reflection intensity was shown. It was demonstrated that ultrasonic measurement is an effective method of detecting wrinkles during press forming.

[doi:10.2320/matertrans.P-M2018807]
\end{abstract}

(Received October 11, 2017; Accepted December 28, 2017; Published March 16, 2018)

Keywords: sheet metal forming, deep drawing, defect, wrinkle, in-process inspection, ultrasonic sensor, sound impedance, relative reflection intensity

\section{Introduction}

The causes of defects that occur during press working vary widely and include nonuniform operation of machines used for processing, wear of metal dies, variation in the amount of lubricant, and subtle changes in tools and workpieces caused by changes in temperature and humidity. These issues have been individually addressed to completely prevent defects. However, the complete prevention of defects is not easy and a defect detection test is carried out at production sites to prevent the outflow of defective products. In the defect detection test of pressed products, the visual inspection and jig inspection of sampled products, rather than all products, are generally carried out in consideration of the cost and labor required for inspection. There is always the risk of an outflow of defective products because not all products are inspected. If defects can be detected when they are generated during processing, the loss associated with defects can be minimized, leading to a quick response to defects and early detection of the cause. From this viewpoint, in-process monitoring of press working has been desired.

There have been several reports on in-process monitoring during press working. Yoshida developed an in-line measuring system using strain gauges, thermocouples, and other devices, enabling the determination of the conditions needed to increase the die lifetime on the basis of various data obtained during hot forging. ${ }^{1)}$ Koyama and Yang incorporated a microsensor fabricated by a technique for processing semiconductors into a die and demonstrated

\footnotetext{
${ }^{* 1}$ This Paper was Originally Published in Japanese in J. JSTP 58 (2017) 393-397.

${ }^{* 2}$ Graduate Student, Kumamoto University

${ }^{* 3}$ Corresponding author, E-mail: y_segawa@cc.miyakonojo-nct.ac.jp
}

that the microsensor can be used in the measurement of the bending angle of workpieces during bending. ${ }^{2)}$ Mahayotsanun et al. proposed a tool-integrated sensing system using two load sensors for deep drawing to optimize the process management. ${ }^{3}$ )

As explained, in-process monitoring during press working becomes possible using various sensors placed at appropriate positions. Measurement using ultrasonic waves has also been used as an effective method for determining the state in dies during press working. We have also examined in-process monitoring of the contact state between a tool and a workpiece during forging using ultrasonic waves. ${ }^{4)}$ StancuNiederkorn et al. proposed a method for simultaneously measuring the real contact area, normal stress, and frictional stress in a ring compression test using ultrasonic waves. ${ }^{5)}$ Hagino and co-workers irradiated products with ultrasonic waves during press working and demonstrated that the amplitudes of reflected and transmitted ultrasonic waves are affected by the material and thickness of workpieces. ${ }^{6,7)}$

If these methods can be applied to the defect detection test, the inspection of all products while suppressing the cost and labor required will be possible. For example, Nishimoto and Shinke evaluated the generation of burrs in punched products using an acoustic emission (AE) sensor and found that the AE energy caused by the generation of burrs is affected by the burr height. ${ }^{8)}$ We have proposed a method for detecting floating scrap generated during the piercing of thin metal sheets using a load sensor and a transmission-type fiber sensor. ${ }^{9)}$ Furthermore, we have developed dies with an embedded ultrasonic probe and found that the reflection characteristics of ultrasonic waves change depending on the state of contact between the dies and the sheet sandwiched between the dies. ${ }^{10)}$ Thus, we have demonstrated the possibility of detecting wrinkles using ultrasonic waves. 


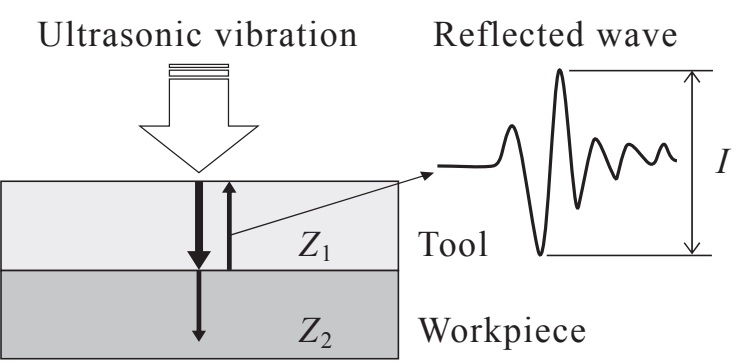

Fig. 1 Schematic illustration of reflection and transmission of ultrasonic wave.

When wrinkles are detected using ultrasonic waves, the relationship between the shape of the wrinkles and the characteristics of the ultrasonic waves should be examined. In this study, workpieces were deep-drawn using a hydraulic forming machine having dies with an embedded ultrasonic probe ${ }^{10)}$ to examine the change in reflection characteristics caused by wrinkling in a flange during deep drawing and the relationship between the shape of wrinkles and the reflection characteristics of ultrasonic waves.

\section{Measurement Principles}

Figure 1 shows a schematic of the reflection and transmission of ultrasonic waves at the interface of a tool and a workpiece in contact with each other. The acoustic impedances $Z=\rho c$ ( $\rho$, density of a medium; $c$, sound velocity in the medium) of the tool and workpiece are $Z_{1}$ and $Z_{2}$, respectively. The ultrasonic waves irradiated from the ultrasonic probe placed above the tool toward the workpiece propagate though the tool and reach the interface between the tool and the workpiece. When $Z_{1}$ and $Z_{2}$ are different, some of the ultrasonic waves are transmitted at the interface and the remaining ultrasonic waves are reflected at the interface. The maximum amplitude of the reflected waves measured with an oscilloscope is defined as reflection intensity $I$.

Figure 2 shows schematics of interfaces in which ultrasonic waves are applied to the detection of wrinkles during sheet forming. When the lower surface of the upper die is in contact with air, as shown in Fig. 2(a), ultrasonic waves are reflected at the lower surface of the upper die. The reflection intensity in this case is denoted as $I_{0}$. When a workpiece is sandwiched between the upper and lower dies, reflection and transmission of ultrasonic waves occur at the interfaces of the tool, workpiece, and lubricant. The reflection intensity in the case of a wrinkle-free workpiece [Fig. 2(d)] is denoted as $I_{3}$. The reflection intensity increases as the contact area between the tool and workpiece decreases with the generation of wrinkles, as shown in Figs. 2(b) and 2(c). In accordance with the decrease in the contact area, the reflection intensity changes $\left[I_{1}\right.$ in Fig. 2(b) and $I_{2}$ Fig. 2(c)]. Here, $I_{0}>I_{1}>I_{2}>I_{3}$. From the change in the reflection intensity, wrinkling and the change in wrinkle shapes can be estimated. In this study, the relative reflection intensity $\left(I / I_{0}\right.$, the ratio of the reflection intensity $I$ at a certain contact state to $I_{0}$ ) was used as the index to evaluate wrinkles.

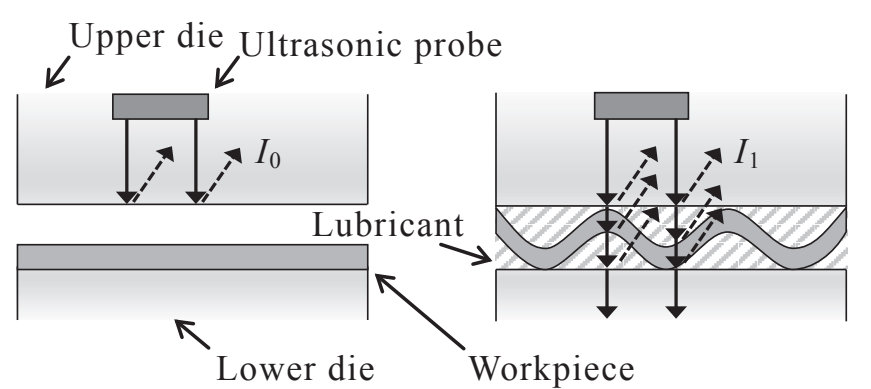

(a)

(b)

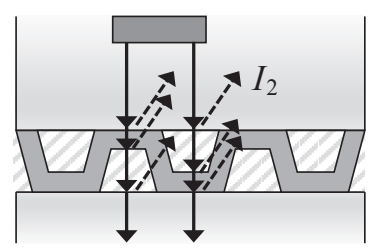

(c)

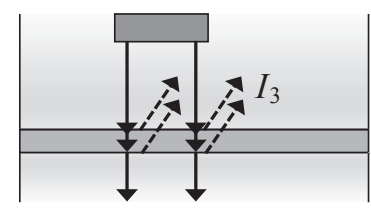

(d)
Fig. 2 Variation in reflection intensity with conditions of wrinkles.

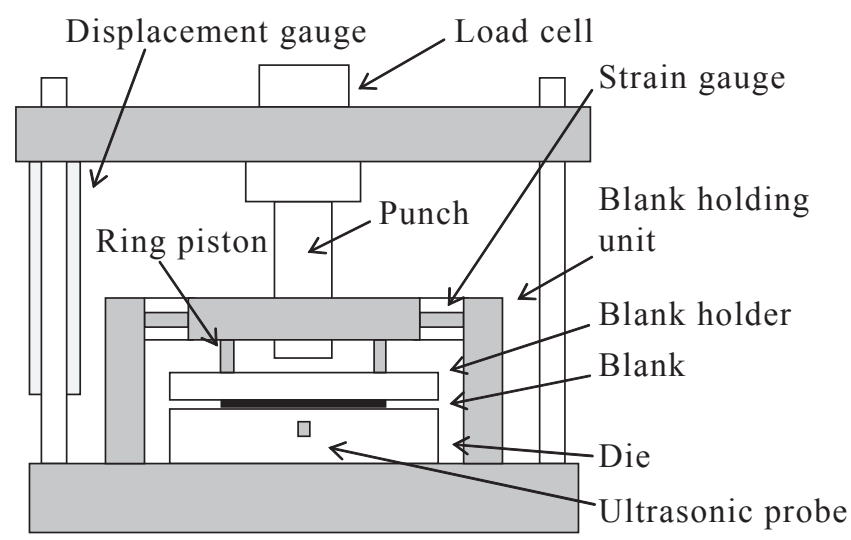

Fig. 3 Schematic illustration of main part of experimental setup.

\section{Evaluation of Wrinkling during Deep Drawing}

\subsection{Experimental setup}

A hydraulic forming machine was used for the evaluation of wrinkling during deep drawing. Figures 3 and 4 show schematics of the hydraulic forming machine and the peripheral equipment connected to it, respectively. In the hydraulic forming machine, the circuits for hydraulic driving of a punch and hydraulic driving of a blank holder were independently operated in different systems. The blank holder force was applied using the ring piston of a blank holder unit and adjusted to a predetermined value during deep drawing. The punch load was detected using a load cell attached above the upper die plate. The blank holder force was detected by strain gauges at the beam of the blank holder unit. The punch stroke was detected with a differential transdisplacement gauge attached to the upper die plate. The data obtained by the above devices were sent to a PC via a data logger. The blank holder force and the workpiece thickness were varied to evaluate the change in the reflection intensity of ultrasonic waves caused by the generation of wrinkles. 


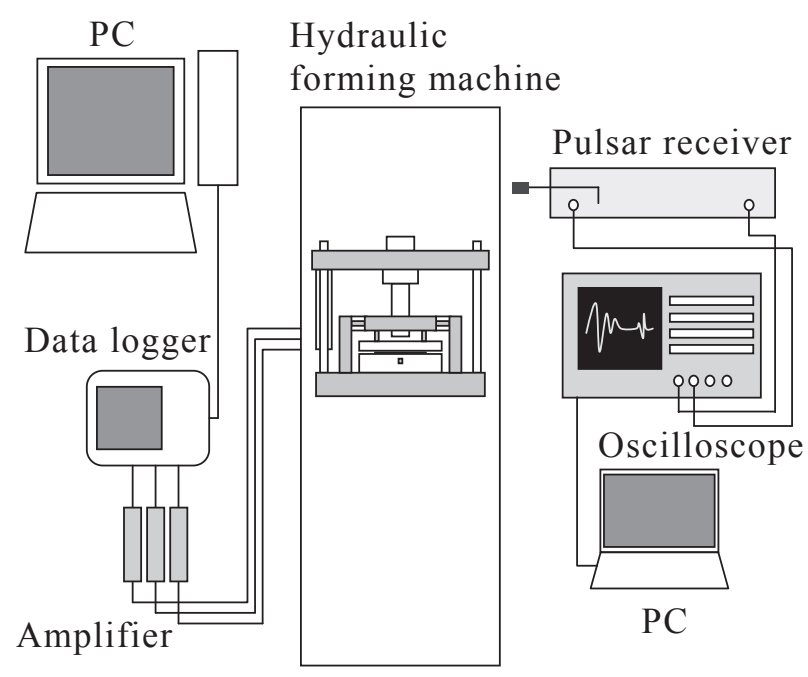

Fig. 4 Hydraulic forming machine and ultrasonic measurement equipment.

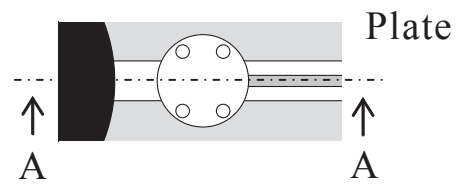

(a) Plan view of lower die

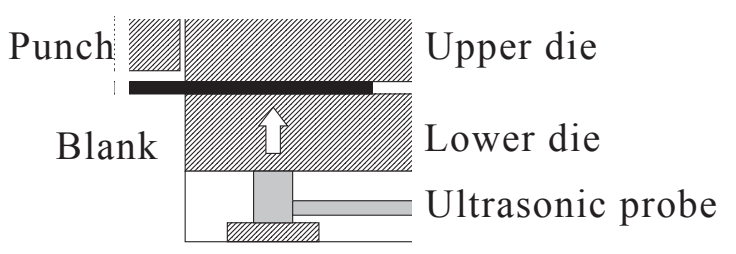

(b) A-A Section

Fig. 5 Installed position of ultrasonic probe.

\subsection{Experimental conditions}

A cylindrical punch with a diameter of $60 \mathrm{~mm}$ was used. The shoulder radius of the punch and die was $4 \mathrm{~mm}$ and the clearance between the punch and die was $1.2 \mathrm{~mm}$. The material of the workpiece was A1050 (thickness $t=0.2,0.3$, and $0.5 \mathrm{~mm}$; drawing ratio, 1.8, 2.0, and 2.2). An ultrasonic probe was embedded in the lower die, ${ }^{10)}$ as shown in Fig. 5, to measure the reflection intensity. The frequency of the ultrasonic waves $f$ was $2.25,5$, and $10 \mathrm{MHz}$ and the effective diameter $D$ was $6.0,6.35$, and $6.5 \mathrm{~mm}$. Ultrasonic waves with a predetermined frequency sent from a pulser receiver (5058PR, Panametrics-NDT) and the ultrasonic probe were transmitted in the die and reflected at the interface between the die and the workpiece. The intensity of the reflected ultrasonic waves was recorded in the PC via the pulser receiver and a digital oscilloscope.

To clarify the relationship between the shape of wrinkles and the reflection intensity, glycerin was spread over the interface between the die and the workpiece. Figure 6 shows the relative reflection intensity, $I / I_{0}$, under four conditions, i.e., with and without glycerin and with and without wrinkles. Without glycerin, almost all ultrasonic waves were reflected regardless of the presence or absence of wrinkles, meaning

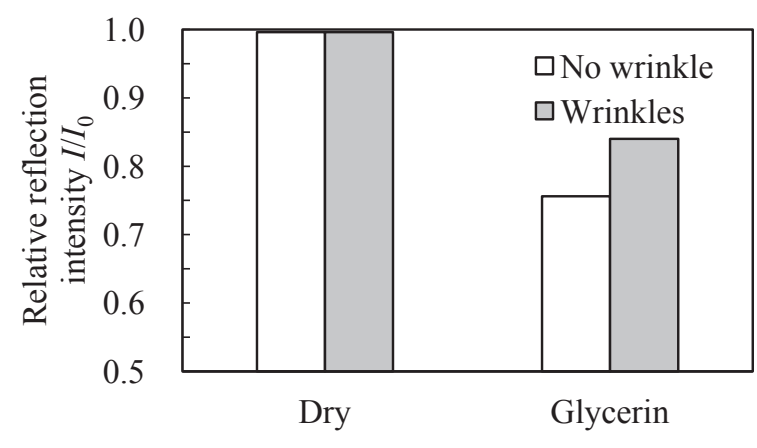

Fig. 6 Influence of using glycerin on relative reflection intensity.

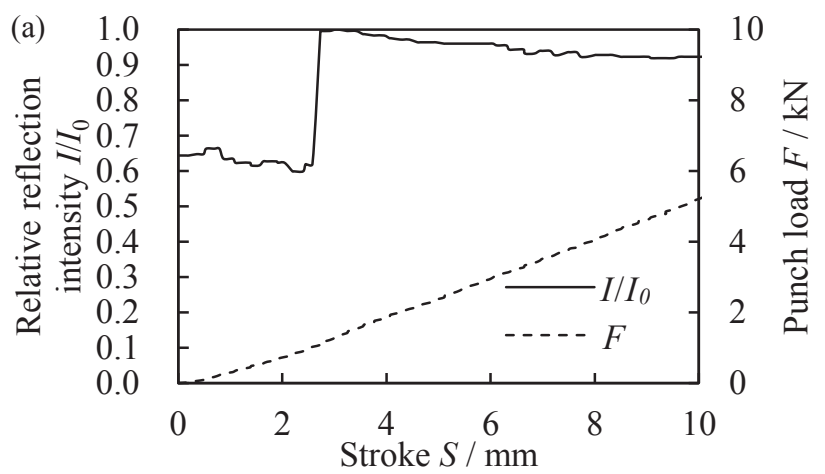

(b)

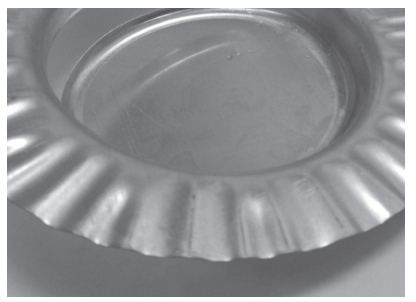

Fig. 7 Results of wrinkle detective experiments in deep drawing process $(t=0.5 \mathrm{~mm}, f=5 \mathrm{MHz}$, Blank holding load $=1.6 \mathrm{kN})$.

that wrinkling was not successfully detected. In contrast, with glycerin, the relative reflection intensity was clearly different with and without the generated wrinkles.

\section{Results and Discussion}

\subsection{Change in relative reflection intensity caused by wrinkling}

Figure 7(a) shows the changes in $I / I_{0}$ and punch load $F$ with punch stroke $S$ during deep drawing when wrinkles with a relatively large height were generated. Figure 7(b) shows a photograph of wrinkles generated in the flange. As shown in Fig. 7(a), $I / I_{0}$ sharply increased from $0.6-0.7$ to $0.9-1.0$ at a stroke of about $2 \mathrm{~mm}$ from the start. At this point, the contact state markedly changed because of wrinkling, leading to an increase in $I / I_{0}$, meaning that most of the ultrasonic waves were reflected.

In contrast, Fig. 8(a) shows the changes in $I / I_{0}$ and punch load $F$ with punch stroke $S$ during deep drawing when minute wrinkles with a small height were generated locally. Figure 8(b) shows a photograph of such wrinkles generated in the flange. As shown in Fig. 8(a), $I / I_{0}$ changed with the stroke; however, no significant change in $I / I_{0}$ was observed. 


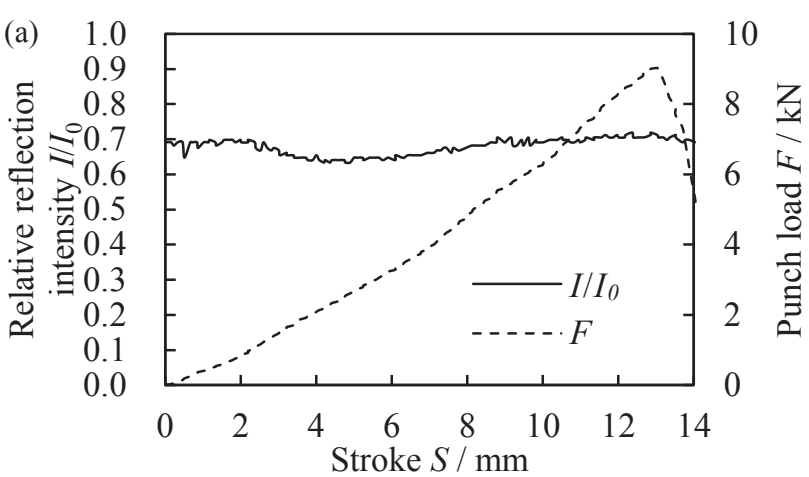

(b)

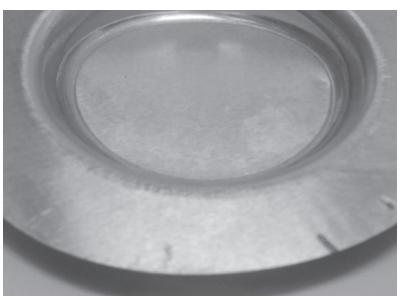

Fig. 8 Results of wrinkle detective experiments in deep drawing process $(t=0.5 \mathrm{~mm}, f=2.25 \mathrm{MHz}$, Blank holding load $=2.8 \mathrm{kN})$.

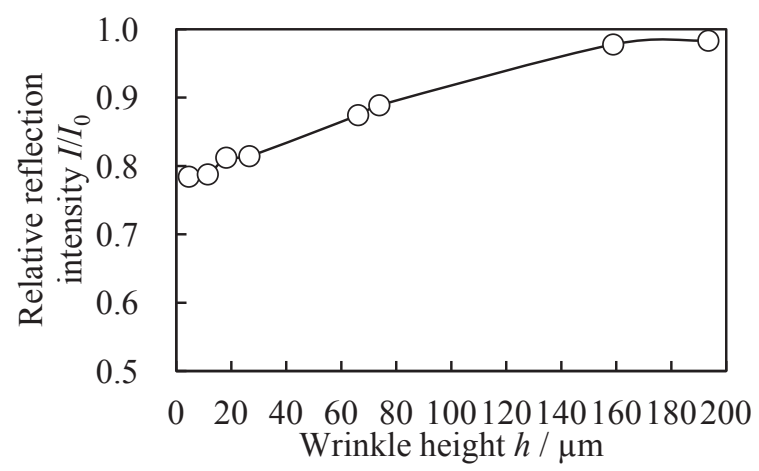

Fig. 9 Relationship between wrinkle height and relative reflection intensity $(t=0.2 \mathrm{~mm}, f=10 \mathrm{MHz})$.

The above findings revealed that wrinkles with a large height generated during deep drawing can be detected with high sensitivity using ultrasonic waves. However, for wrinkles with a small height, the relationship between $I / I_{0}$ and the shape of wrinkles or the frequency of the ultrasonic waves should be clarified first to elucidate the optimal measurement conditions.

\subsection{Relationship between wrinkle height and relative reflection intensity}

Figure 9 shows the relationship between the wrinkle height $h$ and $I / I_{0}$ used to determine the minimum wrinkle height that can be detected. $h$ was measured using a surface roughness measuring instrument (SE-30D, Keyence Corporation). $I / I_{0}$ increased with increasing $h$, meaning that $h$ affected $I / I_{0}$. The change in reflection intensity during drawing was small for wrinkles of $h \leq 30 \mu \mathrm{m}$ and the detection of wrinkles was difficult. In contrast, for wrinkles of $h \geq 70 \mu \mathrm{m}, I / I_{0}$ reached approximately 0.9 . This was significantly different from the reflection intensity before drawing, indicating wrinkling. For very small $h$, the

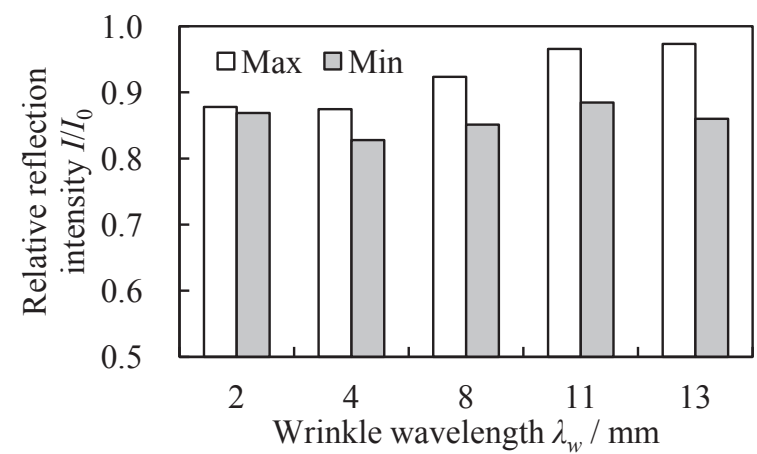

Fig. 10 Relationship between relative reflection intensity and wrinkle wavelength $(f=10 \mathrm{MHz})$.

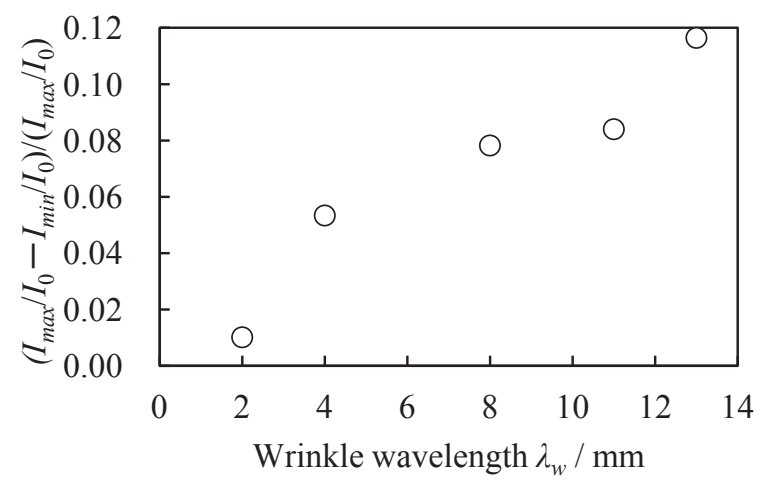

Fig. 11 Relationship between $\left(I_{\max } / I_{0}-I_{\min } / I_{0}\right) /\left(I_{\max } / I_{0}\right)$ and wrinkle wavelength $(f=10 \mathrm{MHz})$.

measurement conditions should be adjusted for wrinkle detection. For relatively large $h$, the detection of wrinkles becomes easier.

\subsection{Effects of wrinkle wavelength and ultrasonic irradiation area}

Reflection intensity is considered to be affected by the ultrasonic irradiation area and the irradiation position of wrinkles. In particular, the effects of wrinkle wavelength $\lambda_{w}$ and ultrasonic irradiation area on $I / I_{0}$ should be examined. Figure 10 shows two types of relative reflection intensity, i.e., maximum value of relative reflection intensity $I_{\max } / I_{0}$ and minimum value of relative reflection intensity $I_{\min } / I_{0}$, with respect to $\lambda_{w}$ obtained under different irradiation positions. At the same $\lambda_{w}, I / I_{0}$ differed depending on the irregularity of wrinkles and the irradiation position. Figure 11 shows the relationship between $\left(I_{\max } / I_{0}-I_{\min } / I_{0}\right) /\left(I_{\max } / I_{0}\right)$ and $\lambda_{w}$. By dividing $I_{\max } / I_{0}-I_{\min } / I_{0}$ by $I_{\max } / I_{0}$, the difference between the maximum and minimum relative reflection intensities becomes a relative value with the effect of $h$ taken into consideration. With increasing $\lambda_{w}$, the difference between the maximum and minimum relative reflection intensities increased.

The above findings were examined with the layouts shown schematically in Figs. 12 and 13. In Fig. 12, the relationship between irradiation distance $L$ and irradiation diameter $D_{1}$ of the ultrasonic probe is shown. When the ultrasonic probe is a round oscillator, the near field length $X_{0}$ and the directional angle $\theta_{0}$ are respectively given by ${ }^{11}$ 


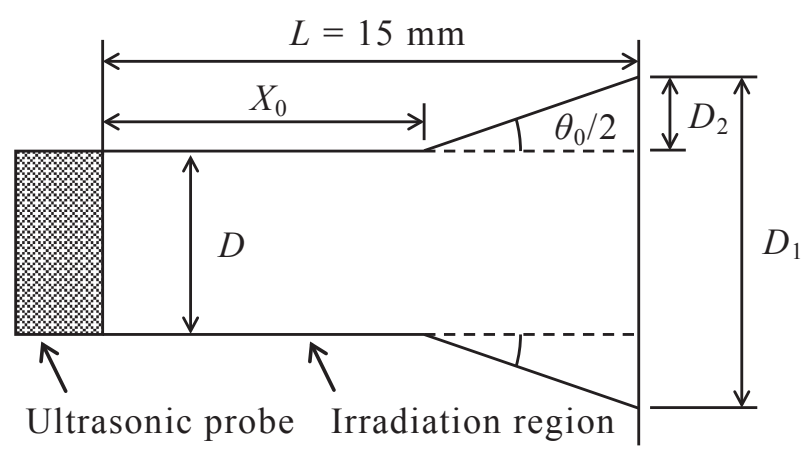

Fig. 12 Relationship between irradiation distance and irradiation diameter.

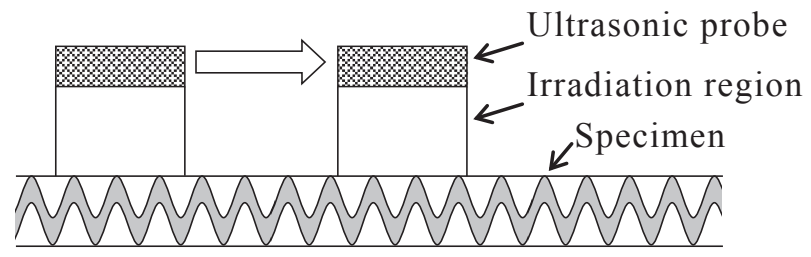

(a) Case of smaller wrinkle wavelength

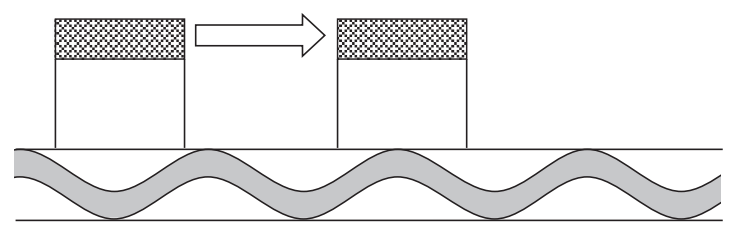

(a) Case of larger wrinkle wavelength

Fig. 13 Relation between wrinkle shape, irradiation diameter and irradiation location

$$
\begin{aligned}
& X_{0}=\frac{D^{2}}{4 \lambda} \\
& \theta_{0}=\frac{70 \lambda}{D}
\end{aligned}
$$

Here, $\lambda$ is the wavelength of ultrasonic waves propagating in a medium. $L$ in this study was $15 \mathrm{~mm}$, as shown in Fig. 12. From eqs. (1) and (2) and Fig. 12, $D_{1}$ was found to be $6.5 \mathrm{~mm}$ for the ultrasonic probe with a frequency of $10 \mathrm{MHz}$. Figure 13 shows the relationship between the ultrasonic irradiation area and wrinkles. When several wrinkles with a small wavelength are included in the ultrasonic irradiation area, as shown in Fig. 13(a), the effect of the wrinkle wavelength on the reflection intensity is small because the contact region in the ultrasonic irradiation area is the same even when the irradiation position moves. In contrast, when the wrinkle wavelength is large, as shown in Fig. 13(b), the effect of the wrinkle wavelength on the reflection intensity is large because the contact region in the ultrasonic irradiation area changes significantly depending on the irradiation position. Therefore, the reflection intensity is considered to vary significantly for large-wavelength wrinkles.

Figure 14 shows the relationship between $\left(I_{\max } / I_{0}-\right.$ $\left.I_{\min } / I_{0}\right) /\left(I_{\max } / I_{0}\right)$ and wrinkle wavelength obtained using an ultrasonic probe with a frequency of $2.25 \mathrm{MHz}$ when the irradiation position was changed. For this ultrasonic probe, $D_{1}$ was $12.1 \mathrm{~mm}$. The reflection intensity was also affected by the wrinkle wavelength and ultrasonic irradiation area when

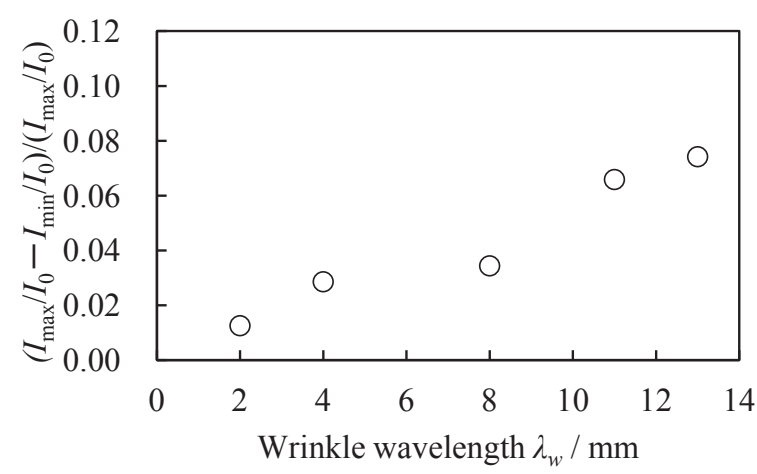

Fig. 14 Relationship between $\left(I_{\max } / I_{0}-I_{\min } / I_{0}\right) /\left(I_{\max } / I_{0}\right)$ and wrinkle wavelength $(f=2.25 \mathrm{MHz})$.

the ultrasonic probe with a frequency of $2.25 \mathrm{MHz}$ was used. However, compared with the case shown in Fig. 11, the difference between the maximum and minimum relative reflection intensities was small at the same $\lambda_{w}$, indicating that the reflection intensity was affected by $D_{1}$.

\section{Conclusion}

Wrinkling in a flange during deep drawing was evaluated using a die with an embedded ultrasonic probe. The following conclusions were obtained.

(1) The wrinkling can be detected as a change in the relative reflection intensity of ultrasonic waves. Therefore, the ultrasonic measurement is effective for in-process monitoring of the wrinkling during deep drawing.

(2) With increasing wrinkle height, the relative reflection intensity increases.

(3) Relative reflection intensity differs depending on the irradiation position even at the same wrinkle wavelength. With increasing wrinkle wavelength, the difference between the maximum and minimum relative reflection intensities increases, indicating that the relationship between the wrinkle wavelength and irradiation diameter affects the relative reflection intensity.

(4) To estimate the optimal measurement conditions for the detection of wrinkles using ultrasonic waves, the effects of the shape of wrinkles, the frequency of ultrasonic waves, and the amount or type of lubricant on the relative reflection intensity should be clarified. We will discuss these effects in detail in our next report.

\section{Acknowledgments}

Part of this study was financially supported by a Japan Society for the Promotion of Science (JSPS) Grant-in-Aid for Scientific Research (C) (Grant Number, 24560139). We would like to thank T. Ma (graduate student, Kumamoto University) and Y. Miyazaki (undergraduate student, Kumamoto University) for their assistance in the experiment.

\section{REFERENCES}

1) T. Yoshida: J. Jpn. Soc. Technol. Plast. 51 (2010) 865-869 (in Japanese).

2) J. Koyama and M. Yang: J. Jpn. Soc. Technol. Plast. 51 (2010) 898- 
902 (in Japanese).

3) N. Mahayotsanun, S. Sah, J. Cao, M. Peshkin, R.X. Gao and C.-T. Wang: Int. J. Mach. Tools Manuf. 49 (2009) 634-644.

4) H. Saiki, Y. Sakata, S. Satonaka, Y. Marumo and Z.H. Zhan: Trans. Jpn. Soc. Mech. Eng., Ser. C 59 (1993) 1934-1939 (in Japanese).

5) S. Stancu-Niederkorn, U. Engel and M. Geiger: J. Mater. Process. Technol. 45 (1994) 613-618.

6) N. Hagino, J. Endou, S. Katoh and M. Ishihama: J. Jpn. Soc. Technol. Plast. 54 (2013) 826-830 (in Japanese).

7) N. Hagino, J. Endou, M. Ishihama, S. Komiya and S. Katoh: Procedia
Eng. 81 (2014) 1073-1078.

8) S. Nishimoto and N. Shinke: J. Jpn. Soc. Non-Destr. Insp. 54 (2005) 557-561 (in Japanese)

9) T. Nonaka, Y. Sakata, Y. Marumo, Y. Okinishi, T. Horinouchi, I. Ogawa and S. Watanabe: Appl. Mech. Mater. 182-183 (2012) 472-476.

10) T. Nonaka, Y. Sakata, Y. Marumo, Y. Okinishi, H. Horinouchi, I. Ogawa and S. Watanabe: Steel Res. Int. Special Edition (2012) 387390.

11) Y. Tanimura: Etoki-Choompa-Tansho-Kiso-No-Kiso (Nikkan-kogyoShimbun-Sha, Tokyo, 2013) pp. 36-39 (in Japanese). 